\title{
Performance comparison of active balancing techniques for lithium-ion batteries
}

\author{
Federico Baronti, Roberto Roncella, Roberto Saletti* \\ Dipartimento di Ingegneria dell'Informazione, University of Pisa \\ Via G. Caruso 16, 56122 Pisa, Italy
}

\begin{abstract}
A simple but effective analysis to calculate the performances achievable by a balancing circuit for series-connected lithium-ion batteries (i.e., the time required to equalise the battery and the energy lost during this process) is described in this paper. Starting from the simple passive technique, in which extra energy is dissipated on a shunt resistor, active techniques, aiming at an efficient energy transfer between battery cells, are investigated. The basic idea is to consider the balancing circuit as a DC/DC converter capable of transferring energy between its input and output with a certain efficiency and speed. As the input and output of the converter can be either a single cell or the entire battery pack, four main active topologies are identified: cell to cell, cell to pack, pack to cell and cell to/from pack (i.e., the combination of the cell to pack and pack to cell topologies when the converter is bidirectional). The different topologies are compared by means of statistical simulations. They clearly show that the cell to cell topology is the quickest and most efficient one. Moreover, the pack to cell topology is the least effective one and surprisingly dissipates more energy than the passive technique, if the converter efficiency is below $50 \%$.

Keywords: Battery equalisation, Battery Management System, Lithium-ion batteries
\end{abstract}

\footnotetext{
* Corresponding author

Email address: r.saletti@iet.unipi.it (Roberto Saletti)
} 


\section{Introduction}

Charge imbalance is a major issue in large-size lithium-ion batteries, in which several cells are series-connected to meet the voltage requirement of the application $[1,2]$. Differences in cell capacity, self-discharge rate and operating temperature cause the charge level to vary from cell to cell. This lack of uniformity in the charge stored in the cells of the battery reduces its usable capacity and lifetime [3]. Charge equalisation is, thus, an important task performed by the Battery Management System (BMS) to provide a safe and effective use of the battery [4]. Different approaches have been investigated to modify the charge level of each cell in a controlled way, in order to bring all the cells to the same charge level at the end of the balancing process [5-11]. They are usually classified into passive and active circuits [12]. The former are only capable of dissipating a controlled amount of energy from each cell of the battery and usually consist of a shunt resistor and a switch per cell. In addition to the intrinsic inefficiency of the method, the balancing speed is limited by the amount of power that can be dissipated in the BMS. Active circuits are more complex and aim at an efficient and fast energy transfer between the cells. In this way, energy is not wasted but moved among the cells to reach charge equalisation. A thorough survey of the different balancing circuits can be found in $[12,13]$.

Those papers also provide a valuable comparison of the different techniques by assigning a "reasonable" mark to various parameters, such as cost, circuit complexity, speed and efficiency. However, these parameters give only a qualitative indication of the performance offered by each technique in balancing the battery, i.e., the balancing time and the energy losses. In fact, not only do these two performance figures depend on the balancing circuit parameters, but also on the strategy that is applied to equalise the battery. Therefore, the comparison presented in $[12,13]$ needs to be completed with a deeper and more quantitative analysis.

The objective of this paper is to extend the analysis carried out in $[12,13]$ by developing a generalised model of various balancing circuits, which allows us 
to derive the optimum balancing strategy for each balancing circuit topology. Optimum balancing means here that battery equalisation is obtained with minimum energy losses. The underlying idea is to represent the balancing circuit as a system capable of transferring energy between its input and output, which are either the cell or the battery terminals. The energy transfer occurs with a certain efficiency and speed, which depend on the circuit implementation, as shown in [13]. As a result, the balancing time and the energy losses of each balancing topology are calculated as a function of the efficiency and speed of the balancing circuit and the initial charge imbalance. Statistical simulations are performed to compare the performance of the different balancing techniques, by generating a large number of random charge imbalances and by evaluating the probability density function (PDF) of the balancing time and energy losses.

This paper is organised as follows. Section 2 describes the generalised model of the different balancing topologies, from which the optimum balancing strategy is derived, as shown in Section 3. Section 4 and 5 describe the comparison methodology and the results of the statistical simulations, respectively. Finally, some conclusions are drawn in Section 6.

\section{Modelling of battery equalisation topologies}

A generic balancing circuit applied to a battery pack consisting of $N$ cells can be seen as an $(N+1)$-port balancing network. As shown in Fig. 1, $N$ ports (cell ports) are connected to the individual cell's terminals and one (pack port) to the terminals of the battery pack. The implementation of the balancing circuit determines the relationship between the ports' currents, and thus how charge is transferred between the battery cells. The voltage at the cell ports is the voltage of the cells $\left(V_{h}, h \in 1 \ldots N\right)$, whereas the overall voltage of the battery $V_{N+1}$ is applied to the pack port. The different balancing circuits can be grouped in five topologies: Cell to Null, Cell to Cell, Cell to Pack, Pack to Cell, and Cell to/from Pack, according to the way by which energy is transferred between the battery cells. Each energy transfer is the result of a DC/DC energy conversion 
characterised by an energy loss and a transfer time, which depend on the efficiency and output power of the DC/DC converter used in the balancing circuit. The aim of this section is to derive an analytical model for each topology, which allows the computation of the balancing performances that can be achieved.

To this end, the port currents $I_{j}, j \in 1 \ldots N+1$ of the balancing network will be related to the parameters of the $\mathrm{DC} / \mathrm{DC}$ converter and to the control strategy of the balancing circuit, which equalises the battery with minimum loss of energy. We neglect the dynamic behaviour of the battery and the dependence of the cell open circuit voltage $O C V$ on the state-of-charge $S o C$ [14]. In fact, the cell voltage is considered constant and equal to its average value $\bar{V}$ in the $\mathrm{SoC}$ range identified by the lowest and the most charged cell in the pack. This approximation leads to simply modelling the balancing network, as the charge flowing through each port of the network depends only on the DC/DC converter parameters, being the port voltages constant. A simple model allows us to derive analytical expressions for the balancing performances achieved by the different topologies, thus making their quantitative comparison possible. It is important to note that the constant cell voltage approximation is acceptable because the balancing currents are typically much smaller than the cell C-rate, the slope of the $O C V-S o C$ curve is rather low, particularly in some kinds of batteries, and the maximum $S o C$ range in which the assumption must hold is usually small (e.g., below $10 \%$ ). This last hypothesis is a direct consequence of the availability of a balancing circuit in the BMS. Moreover, we assume that the $\mathrm{DC} / \mathrm{DC}$ converter operates in constant current mode and with constant efficiency. The five balancing topologies and their models are presented and discussed in the following.

- Cell to Null (C2N, or passive balancing): Energy is selectively extracted from any cell and dissipated in a shunt resistor, until all the cells reach the same charge level. The balancing network is modelled with $N$ zero-efficiency DC/DC converters, the input of each is a cell port of the balancing network. The currents flowing in the ports of the balancing 
network are

$$
I_{j}= \begin{cases}I_{\mathrm{sh}}, & \text { if cell } j \text { is selected } \\ 0 & \text { if cell } j \text { is deselected or } j=N+1\end{cases}
$$

where $I_{\mathrm{sh}}$ is the current through the shunt resistor $R . I_{\mathrm{sh}}$ can be considered constant for the assumptions made $\left(I_{\mathrm{sh}}=\bar{V} / R\right)$. Practical values of $I_{\mathrm{sh}}$ are in the order of hundreds of milliamperes and are bounded by the maximum power that can be dissipated in the BMS.

- Cell to Cell (C2C): Two cells are selected for the energy transfer. Energy is extracted from one cell and delivered to the other. Then, the operation is sequentially repeated on another pair of cells, until all the cells reach the same charge level. The balancing network is modelled with a single $\mathrm{DC} / \mathrm{DC}$ converter, whose input and output are the ports corresponding to the selected cells. If $h$ and $k(h, k \in 1 \ldots N$ and $h \neq k)$ are the ports connected to the converter input and output respectively, it follows that

$$
I_{j}= \begin{cases}-I_{\mathrm{bal}}, & j=k \\ \frac{I_{\mathrm{bal}} V_{k}}{\eta V_{h}} \simeq \frac{I_{\mathrm{bal}}}{\eta}, & j=h \\ 0 & j \neq h \text { and } k\end{cases}
$$

where $I_{\text {bal }}$ is the constant output current of the converter (usually from hundreds of milliamperes to a few amperes) and $\eta$ is its efficiency.

- Cell to Pack (C2P): One cell is selected. Energy is extracted from it and equally delivered to all the cells through the pack's terminals, i.e., the port $N+1$. The balancing network is modelled with a single DC/DC converter, whose input is the selected cell port and its output is the pack port. If $h$ $(h \in 1 \ldots N)$ is the port selected as the converter input, it follows that

$$
I_{j}= \begin{cases}I_{\mathrm{bal}}, & j=h \\ -\frac{\eta I_{\mathrm{bal}} V_{h}}{V_{N+1}} \simeq-\frac{\eta I_{\mathrm{bal}}}{N}, & j=N+1 \\ 0 & j \neq h \text { and } N+1\end{cases}
$$


where $I_{\text {bal }}$ is the constant input current of the converter (usually from hundreds of milliamperes to a few amperes) and $\eta$ is its efficiency.

- Pack to Cell (P2C): One cell is selected. Energy is equally extracted from all the cells through the pack's terminals and delivered to the selected cell. The balancing network is modelled with a single DC/DC converter, whose input is the pack port (port $N+1$ ) and its output is the selected cell port. If $h(h \in 1 \ldots N)$ is the port selected as the converter output, it follows that

$$
I_{j}= \begin{cases}-I_{\mathrm{bal}}, & j=h \\ \frac{I_{\mathrm{bal}} V_{h}}{\eta V_{N+1}} \simeq \frac{I_{\mathrm{bal}}}{\eta N}, & j=N+1 \\ 0 & j \neq h \text { and } N+1\end{cases}
$$

where $I_{\text {bal }}$ is the constant output current of the converter (usually from hundreds of milliamperes to a few amperes) and $\eta$ is its efficiency.

- Cell to/from Pack (C2P2C): Both energy transfers (Cell to Pack and Pack to (ell) are implemented. The balancing network is modelled as the Cell to Pack or the Pack to Cell methods, depending on the direction of the energy transfer. We assume that the bidirectional DC/DC converter has the same efficiency in both directions and that the input current in the Cell to Pack direction is equal to the output current in the Pack to Cell direction.

\section{Battery equalisation algorithms}

The model of each balancing topology described in Section 2 makes it possible to develop the related best balancing algorithm, which minimises the energy lost by the battery to recover its balanced condition. To this aim, let us define $\mathrm{Qbal}_{j}=\int_{T} I_{j} \mathrm{dt}, j \in 1 \ldots N+1$, as the charges entering each port of the balancing network during the balancing time $T$, after which the battery is balanced, i.e., all the cells store the same charge $Q_{\mathrm{end}}$. Say $Q_{h}$ the charge stored in the cell $h, h \in 1 \ldots N$ before balancing. $Q_{\text {end }}$ is reached by each cell at the 
end of balancing after having individually exchanged the charge $Q b a l_{h}$ through its cell port, and globally exchanged the charge $Q b a l_{N+1}$ through the pack port. Therefore, the following relationship holds.

$$
Q_{\mathrm{end}}=Q_{h}-Q_{b a l_{h}}-Q b a l_{N+1}, h \in 1 \ldots N
$$

Our aim is to calculate the values $Q_{b a l}$ that maximise $Q_{\text {end }}$ and thus minimise the overall energy $E_{\text {loss }}$ lost by the battery during balancing. $E_{\text {loss }}$ is the difference between the battery energy before and after balancing, expressed as:

$E_{\text {loss }}=\bar{V} \sum_{h=1}^{N} Q_{h}-N \bar{V} Q_{\text {end }}=\bar{V} \sum_{h=1}^{N}\left(Q_{h}-Q_{\text {end }}\right)=\bar{V}\left(\sum_{h=1}^{N} \operatorname{Qbal}_{h}+N \operatorname{Qbal}_{N+1}\right)$

Before proceeding to the calculation of $Q_{\text {end }}$ and $T$ for each topology, let us sort the cells in descending order by their charge level before balancing, i.e., $Q_{1} \geq Q_{2} \geq \cdots \geq Q_{N}$

- Cell to Null: As energy is only extracted from the cells, the optimum way to reach the balance is by discharging all the cells to the minimum level $Q_{N}$. Assuming that all the cells are discharged in parallel, the balancing time is the time needed to bring the maximum charge level $Q_{1}$ down to $Q_{N}$. From (1),

$$
\begin{aligned}
& Q_{\text {end }}=Q_{N} \\
& T=\frac{Q_{1}-Q_{\text {end }}}{I_{\text {sh }}}
\end{aligned}
$$

- Cell to Cell: Minimising $E_{\text {loss }}$ implies minimising the charge transferred between the cells, as each transfer involves an energy loss. This means that each cell charge level must reach $Q_{\text {end }}$ monotonically. This results in finding the number $M$ of the more charged cells that provide charge to the $N-M$ less charged ones, i.e., finding $Q_{\text {end }}$ so that $Q_{M} \geq Q_{\text {end }} \geq$ $Q_{M+1}$. Recalling the equations (2), $Q_{\text {end }}$ and $M$ must also satisfy the following relationship, which links the charge extracted from the $M$ more charged cells, the charge delivered to the $N-M$ less charged ones, and 
the converter efficiency.

$$
\eta \sum_{h=1}^{M}\left(Q_{h}-Q_{\text {end }}\right)=\sum_{h=M+1}^{N}\left(Q_{\text {end }}-Q_{h}\right)
$$

which leads to

$$
\left\{\begin{aligned}
Q_{\mathrm{end}} & =\frac{\eta \sum_{h=1}^{M} Q_{h}+\sum_{h=M+1}^{N} Q_{h}}{N-M(1-\eta)} \\
Q_{M} \geq Q_{\mathrm{end}} & \geq Q_{M+1}
\end{aligned}\right.
$$

Note that $Q_{\text {end }}$ is the average value of the charge levels before balancing, if $\eta=1$ (no energy is lost in the transfers). Finally, the balancing time can be calculated as the charge delivered to the $(N-M)$ less charged cells divided by the output current of the converter

$$
T=\frac{\sum_{h=M+1}^{N}\left(Q_{\mathrm{end}}-Q_{h}\right)}{I_{\mathrm{bal}}}
$$

- Cell to Pack: As in the Cell to Null method, the optimum way to reach the condition in which all the cells are balanced is to extract the balancing charge $Q_{b a l}=Q_{h}-Q_{N}, h \in 1 \ldots N$ from each cell. This charge is then globally redistributed to every cell of the pack, instead of being dissipated. Actually, the Cell to Null method is a particular case of the Cell to Pack topology, when the efficiency of the converter is set to zero. Therefore, $Q_{\text {end }}$ is always greater than the minimum charge level $Q_{N}$, if $\eta>0$. Using (3), we obtain

$$
\begin{aligned}
& Q_{\text {end }}=Q_{N}+\frac{\eta}{N} \sum_{h=1}^{N}\left(Q_{h}-Q_{N}\right) \\
& T=\frac{\sum_{h=1}^{N} Q b a l_{h}}{I_{\text {bal }}}=\frac{\sum_{h=1}^{N}\left(Q_{h}-Q_{N}\right)}{I_{\text {bal }}}
\end{aligned}
$$

- Pack to Cell: This method is complementary to the Cell to Pack method. In fact, the optimum way to reach the balanced condition is to deliver the individual balancing charges $\left|Q_{b a l_{h}}\right|=Q_{1}-Q_{h}, h \in 1 \ldots N$ to the cells (we recall that the balancing charge is positive when extracted from 
a cell), at the expense of a charge globally extracted from the pack. Using (4), we obtain

$$
\begin{aligned}
& Q_{\text {end }}=Q_{1}-\frac{1}{\eta N} \sum_{h=1}^{N}\left(Q_{1}-Q_{h}\right) \\
& T=\frac{\sum_{h=1}^{N}\left|Q b a l_{h}\right|}{I_{\text {bal }}}=\frac{\sum_{h=1}^{N}\left(Q_{1}-Q_{h}\right)}{I_{\text {bal }}}
\end{aligned}
$$

Note that $Q_{\text {end }}$ can even be less than $Q_{N}$ depending on the converter efficiency and the actual charge imbalance.

- Cell to/from Pack: The possibility to independently deliver or extract charge to/from any cell provides a degree of freedom in the choice of the balancing charges that equalise the battery. $Q_{b a l}$ is indeed the sum of two components: $Q b a l_{h}^{C 2 P}$ and $Q_{b a l}^{P 2 C}$, i.e., the charge entering the cell port $h$ when the converter direction is Cell to Pack and Pack to Cell, respectively $\left(Q_{b a l}^{C 2 P}\right.$ is a non negative value and $Q b a l_{h}^{P 2 C}$ is a non positive value). To achieve battery balancing, the two components $Q_{b a l}^{C 2 P}$ and $\mathrm{Qbal}_{h}^{P 2 C}$ must satisfy the following relationship for each cell $(h \in 1 \ldots N)$.

$$
Q_{\text {end }}=Q_{h}-Q b a l_{h}^{C 2 P}-Q b a l_{h}^{P 2 C}-\frac{1}{N} \sum_{j=1}^{N}\left(-\eta Q b a l_{j}^{C 2 P}-\frac{Q b a l_{j}^{P 2 C}}{\eta}\right)
$$

The last term in (13) is the charge entering the pack port in Fig. 1 and is the same for all the cells. Thus $Q_{b a l}$ can be computed using (14) for each cell, where $Q^{*}$ is a generic charge value between $Q_{N}$ and $Q_{1}$.

$$
Q_{b a l}=Q_{h a l}^{C 2 P}+Q_{h}^{P a l}{ }_{h}^{P 2 C}=Q_{h}-Q^{*}
$$

The aim is to find $Q b a l_{h}^{C 2 P}$ and $Q b a l_{h}^{P 2 C}(h \in 1 \ldots N)$ that maximise $Q_{\text {end }}$, which is equivalent to minimise $E_{\text {loss }}$, according to (6). Eq. (14) implies that only one of the two converter directions must be used, according to the sign of $\mathrm{Qbal}_{h}$, in order to minimise the energy lost to equalise each cell (and thus the overall battery). This means that $Q b a l_{h}=Q b a l_{h}^{C 2 P}$ if $Q_{b a l_{h}} \geq 0$ or $Q_{b a l}=Q_{h} b a l_{h}^{P 2 C}$ otherwise. As the cells are sorted by the 
charge level in descending order, it is possible to find the cell $M$ for any given value of $Q^{*}$, so that $Q_{M} \geq Q^{*} \geq Q_{M+1}$. The Cell to Pack direction is then applied only to the $M$ more charged cell, while the Pack to Cell to the $(N-M)$ less charged ones. Eq. (13) can be rewritten as follows

$$
Q_{\text {end }}=Q^{*}+\frac{\eta}{N} \sum_{j=1}^{M}\left(Q_{j}-Q^{*}\right)-\frac{1}{\eta N} \sum_{j=M+1}^{N}\left(Q^{*}-Q_{j}\right)
$$

where $Q_{\text {end }}$ is a function of $M$ and $Q^{*}$, with $Q_{M} \geq Q^{*} \geq Q_{M+1}$. Eq. (15) yields that $Q_{\text {end }}$ is maximum when $Q^{*}=Q_{M+1}$. Substituting this value in (15), we eventually find the value of $M$ that maximises $Q_{\mathrm{end}}$, i.e., Mopt $=\lfloor N /(1+\eta)\rfloor$. It is worth noting that Mopt depends only on the converter efficiency and the number of cells and not on the actual charge imbalance. The expressions for $Q_{\text {end }}$ and the balancing time $T$ are as follows

$$
\begin{aligned}
& Q_{\text {end }}=Q_{M o p t+1}+\frac{\eta}{N} \sum_{j=1}^{M o p t}\left(Q_{j}-Q_{M o p t+1}\right)-\frac{1}{\eta N} \sum_{j=M o p t+1}^{N}\left(Q_{M o p t+1}-Q_{j}\right) \\
& T=\frac{\sum_{h=1}^{N} Q b a l^{C 2 P}+\left|Q b a l^{P 2 C}\right|}{I_{\text {bal }}}=\frac{\sum_{h=1}^{N}\left|Q_{M o p t+1}-Q_{h}\right|}{I_{\text {bal }}}
\end{aligned}
$$

where Mopt $=\lfloor N /(1+\eta)\rfloor$.

For every balancing method, we have finally obtained the analytical expressions that allow the calculation of the energy losses and the balancing time, for any given distribution $Q_{h}, h \in 1 \ldots N$ of the charges in the battery cells, as a function of the balancing current and the converter efficiency. This result is the core of the quantitative comparison methodology described in the following Section.

\section{Comparison methodology}

The aim of this section is to define a methodology to compare the different balancing topologies, given the parameters of the balancing circuits that implement them. The basic idea is to perform a statistical experiment, consisting in randomly generating an initial unbalanced charge distribution in the battery. 
Then, the algorithms described in Section 3 to evaluate the balancing metrics (i.e., the energy losses and the balancing time) are applied. We note that each cell port of the balancing network model is equivalent to any other, thus the balancing metrics do not depend on which cell of the pack is connected to a certain cell port.

The following assumptions are made to carry out the comparison:

- $Q_{\max }$ is the maximum charge that can be stored in any cell.

- $Q_{1}=Q_{\max }$ and $Q_{N}=(1-\delta) Q_{\max }, \delta \in[0,1]$, where $\delta$ is the maximum SoC mismatch between the cells.

- $Q_{h}=\left(1-\alpha_{h}\right) Q_{\max }, h \in 2 \ldots N-1$, where $\alpha_{h}$ are independent random variables uniformly distributed in $[0, \delta]$.

The above assumptions mean that one cell is considered fully charged in each experiment trial. The maximum $S o C$ mismatch is always equal to $\delta$ and is due to the $N$-th cell, whereas the $S o C$ of the remainder $N-2$ cells varies in between for each experiment trial. These assumptions are made considering that the balancing procedure usually starts at the end of battery charging, when the most charged cell reaches the charging cut-off voltage. However, the experiments could be carried out for any given choice of the $Q_{1}$ and $Q_{N}$ values.

The comparison of the five balancing topologies is carried out by taking the passive method ( Cell $2 \mathrm{Null}$ ) as a reference. Therefore, we define two comparison parameters, the balancing time figure $F_{\text {time }}$ and the energy loss figure $F_{\text {loss }}$, as the balancing time and energy loss for each topology divided by the respective values calculated for the Cell 2 Null one. $F_{\text {time }}$ and $F_{\text {loss }}$ do not depend on $Q_{\max }$, as the balancing time and energy loss are proportional to $Q_{\max }$ for all the methods. $F_{\text {time }}$ and $F_{\text {loss }}$ are, in their turn, random variables. It is worth noting that finding a value of these variables less than one in an experiment trial means a better performance of the active technique compared to the passive one in that particular trial.

As a case study, the comparison methodology described above has been 
applied to a battery pack consisting of $N=10$ lithium iron phosphate $\left(\mathrm{LiFePO}_{4}\right)$ cells. This configuration is common in both medium power applications (like an e-bike, where only one battery pack is used) and higher power applications (like electric vehicles, where more battery packs are series-connected to reach the required battery voltage level). Figure 2 shows the typical $S o C-O C V$ of the $\mathrm{LiFePO}_{4}$ battery chemistry, as experimentally measured in our laboratory. It is worth noting that considering the $O C V$ constant when $70 \% \leq S o C \leq 100 \%$ is a well justified approximation. Indeed, the constant voltage approximation $\bar{V}=3.344 \mathrm{~V}$ leads to an error below $1 \%$ with respect to both the charge and discharge $S o C-O C V$ curves of the battery. A larger error is expected for other battery chemistry that show less flat $S o C-O C V$ curves. However, it should be reminded that only a minor part of the entire $S o C$ range is involved, so that the error induced by the constant cell voltage assumption can still be considered acceptable.

Further, we assume the maximum $S o C$ mismatch $\delta=0.1=10 \%$ and the same converter efficiency and balancing current for all the four active balancing topologies. In particular, $\eta=0.85, I_{\mathrm{bal}}=1 \mathrm{~A}$, and $I_{\mathrm{sh}}=200 \mathrm{~mA}$, if not specified otherwise in the following. These are reasonable practical values for the $\mathrm{DC} / \mathrm{DC}$ converter and the shunt resistor implementing the active balancing method and the Cell to Null one, respectively [8, 15]. It should be noted that the balancing time $T_{\mathrm{bal}}^{C 2 N}$ of the passive method is constant, as it is derived from (7)

$$
T_{\mathrm{bal}}^{C 2 N}=\frac{Q_{1}-Q_{N}}{I_{\mathrm{sh}}}=\frac{\delta Q_{\max }}{I_{\mathrm{sh}}}
$$

On the contrary, the balancing time of the active methods and the energy loss of all the methods are a function of the initial charge distribution. From (11) and (12), we can easily calculate the mean value of the balancing times $\left\langle T_{\mathrm{bal}}^{C 2 P}\right\rangle$ and $\left\langle T_{\mathrm{bal}}^{P 2 C}\right\rangle$ for the Cell to Pack and Pack to Cell methods, respectively.

$$
<T_{\text {bal }}^{C 2 P}>=<T_{\text {bal }}^{P 2 C}>=\frac{N \delta Q_{\text {max }}}{2 I_{\text {bal }}}
$$

These values are equal to each other. They are also equal to $T_{\mathrm{bal}}^{C 2 N}$, the passive method balancing time expressed in (17), if $I_{\text {bal }}=I_{\mathrm{sh}} N / 2$, as we have chosen in 
our case study.

\section{Comparison results}

In order to compare the performances of the five balancing topologies considered, the probability density function $(\mathrm{PDF})$ of the random variables $F_{\text {time }}$ and $F_{\text {loss }}$ were estimated by repeating 100,000 times the above described experiment (with the parameters summarised in Table 1). The estimated PDFs are shown in Fig. 3 and 4. It should be noted that the Cell to Cell and the Cell to/from Pack clearly outperforms the other methods in terms of both balancing time and energy loss.

If we first consider the balancing time (Fig. 3), the Cell to Pack and the Pack to Cell statistically behaves in the same way, as it is expected from (11), (12) and the uniform distribution of the cell charges. Since the PDFs are symmetric around 1, these methods behave on average as the Cell to Null one, as expected from (18) and the choice of $I_{\text {bal }}$ and $I_{\text {sh. }}$. Instead, the Cell to Cell and Cell to/from Pack methods allow an average reduction of the balancing time of a factor 3.8 and 1.8, respectively. This reduction is expected because the time to balance the battery depends, at the same balancing current, on how much charge has to be moved. The cell optimum balancing charge is computed as the difference between the actual cell level and either the highest or the lowest level for the Pack to Cell, Cell to Null and Cell to Pack methods. Instead, an intermediate charge level is used in the Cell to Cell and Cell to/from Pack methods. Less charge to be transferred implies a smaller balancing time.

The same reasoning also justifies the performance of the four active balancing topologies for what concerns the energy losses, which are different even if the DC/DC converters have the same efficiency, as shown in Fig. 4. In particular, for $\eta=0.85$, the energy loss for the Cell to Cell, Cell to/from Pack, Cell to Pack, and Pack to Cell topologies is on average reduced, when compared to the Cell to Null one, by a factor around $21,10.9,6.7$, and 5.3 , respectively. $F_{\text {loss }}$ for the

Cell to Pack topology does not depend on the statistical experiment outcome, as the related energy losses are equal to $(1-\eta)$ time those of the Cell to Null 
topology. Thus, the PDF for the Cell to Pack method is a Dirac delta function (with unity area) positioned in $(1-\eta)=0.15$, as shown in Fig. 4. Further, the Pack to Cell performs slightly worse than the Cell to Pack, despite the same balancing charges on average. This is because delivering a given amount of charge to a cell implies a higher loss of energy than extracting the same amount of charge from a cell. This is just what happens in comparing the Pack to Cell to the Cell to Pack method, where the same balancing charges are in the first case delivered to cells and in the second extracted from them.

An important conclusion is that all the four active balancing topologies well outperform the passive one, given the relatively high efficiency of the converter $(\eta=0.85)$. It is now interesting to show how much the balancing performance comparison depends on the converter efficiency. This behaviour is clearly visible in Fig. 5, where the mean value $\left\langle F_{\text {loss }}>\right.$ of $F_{\text {loss }}$ is plotted as a function of the converter efficiency for the four active methods (the mean value has been calculated from 10,000 trials). Active balancing is always better than passive except for the Pack to Cell topology. Indeed, when the converter efficiency goes below 0.5 , we come to the surprising conclusion that active balancing can be worse than passive, as the energy losses are on average larger. This result is confirmed by the analytical expressions of the Cell to Null and Pack to Cell average losses calculated from (6), (7) and (6), (12) respectively, and shown below.

$$
\begin{gathered}
<E_{\text {loss }}^{C 2 N}>=\frac{N \delta Q_{\max }}{2} \bar{V} \\
<E_{\text {loss }}^{P 2 C}>=<E_{\text {loss }}^{C 2 N}>\frac{1-\eta}{\eta}
\end{gathered}
$$

It is worth noting that, given the converter efficiency, the Cell to Cell topology behaves significantly better than the other methods, even for low values of the efficiency. For instance, the use of a low performance converter with a low efficiency of 0.5 still allows the reduction of the energy losses on average to 
one fifth with respect to passive balancing. We can finally draw the conclusion that, besides the converter efficiency, the most important factor to improve the efficiency in balancing a battery is the method by which balancing is achieved.

Finally, we analyse the power $P_{\text {loss }}$ dissipated by the balancing circuits during battery equalisation. $P_{\text {loss }}$ is due to the losses in the DC/DC converter in the active techniques. It can directly be calculated from the converter efficiency for the Cell to Cell, Cell to Pack and Pack to Cell topologies and is equal to $V I_{\text {bal }}(1-\eta) \cong 0.5 \mathrm{~W}$, for the first two topologies, and to $V I_{\text {bal }}(1-\eta) / \eta \cong 0.59 \mathrm{~W}$ for the third one ( $\eta=0.85$ in our case study). As the Cell to/from Pack topology is the combination of the Cell to Pack and the Pack to Cell topologies, $P_{\text {loss }}$ assumes one of the above values, according to the converter direction set by the optimum control strategy derived in Section 3. However, these two values are close to each other for relatively high efficiency values. Thus, $P_{\text {loss }}$ can be considered constant during the balancing time and independent of the charge imbalance for all the four active topologies.

On the other hand, the power dissipated in the Cell to Null topology strongly depends on the actual charge distribution. The latter determines the overall energy to be dissipated by the shunt resistors, which varies in each trial, whereas the balancing time [see (17)] is constant in our statistical experiment. If we define $P_{\text {loss }}=E_{\text {loss }} / T_{\text {bal }}$ for the Cell to Null topology, we can evaluate its PDF, which is shown in Fig. 6. The mean value of $P_{\text {loss }}$ can be calculated from (5) and (17) and is $N I_{\mathrm{sh}} \bar{V} / 2=3.334 \mathrm{~W}$. However, the BMS must be designed to dissipate the maximum value of $P_{\text {loss }}$, i.e., $(N-1) I_{\mathrm{sh}} \bar{V}=6 \mathrm{~W}$, when all the shunt resistors, apart one, are enabled. This value is ten times higher than that required by the active topologies and might exceed the maximum power $P_{\max }$ that can be dissipated by the BMS. In this case, all the needed shunt resistors cannot simultaneously be enabled, as the $P_{\max }$ limit would be exceeded. Some of the shunt activations have to be delayed and thus the balancing time increases. The latter is indeed no longer determined by the maximum charge imbalance only, but depends also on $E_{\text {loss }}$, which cannot be dissipated at a rate greater than $P_{\max }$. The exact calculation of the balancing time is relatively complex when 
only a subset of the shunt resistors can simultaneously be enabled. However, a good approximation can be obtained by choosing the maximum between the value given by (17) and $E_{\text {loss }} / P_{\max }$. In fact, the balancing time can even be longer, because the number of shunt resistors that need to be enabled might be less than the maximum allowed (usually towards the end of of the balancing time, where a few cells still need to be discharged). Thus, the power dissipated by the shunt resistors is not constant during all the balancing time and this depends on the charge distribution among the cells.

If we now compute the balancing time figure also in this case, we obtain the plot shown in Fig. 7, which shows how much the mean value of the balancing time increases when the power dissipated is constrained to $P_{\max }$. It is worth noting that if $P_{\max }$ is equal to the mean value of $P_{\text {loss }}\left(P_{\max }=3.334 \mathrm{~W}\right.$, i.e., only up to half of the shunt resistors can be enabled simultaneously), the balancing time increases on average by only $7 \%$. To give an idea of the time required for passive balancing, which is our reference value, let us consider again the case study battery consisting of $40 \mathrm{Ah}$ cells. According to (17), the balancing time is $20 \mathrm{~h}$ in the best case, with no constraints on the dissipated power. If only one shunt resistor is enabled at a time, which means that the power dissipated is roughly the same as in the active topologies, the balancing time increases on average by a factor of 5 , reaching the impractical value of $100 \mathrm{~h}$.

\section{Conclusions}

We have first developed an effective analysis to evaluate the performances of the most common balancing circuits used to equalise a battery consisting of series-connected cells. This analysis extends recently published results. Five basic topologies have been considered, i.e., Cell to Null, Cell to Cell, Cell to Pack, Pack to Cell, and Cell to/from Pack. Each topology is modelled by the type of the energy transfer, the speed and efficiency at which the transfer occurs. This model has allowed us to compute the minimum balancing time and energy losses achievable by each topology, given the initial charge imbalance. Then, the five topologies have been compared by means of statistical simulations. Passive 
balancing (i.e., Cell to Null topology) has been used as reference to compare the four active topologies, given the same balancing current and efficiency.

The direct energy transfer from cell to cell (Cell to Cell topology) outperforms all the other topologies in terms of both energy losses and balancing time. An effective application of this technique is shown in [15]. It is worth noting that the model of Cell to Cell topology implies that the energy transfer occurs between any given couple of cells. Thus, the techniques based on switching capacitors [5], where charge can only be transferred between adjacent cells proportionally to the cell voltage difference, are not included in the model. However, these techniques are not particularly effective for lithium-ion batteries, where the $S o C-O C V$ curve is almost flat in a wide range of $S o C$. Cell to/from Pack, Cell to Pack and Pack to Cell perform in this order. In fact, the Pack to Cell topology, whose typical implementation is based on a flyback DC/DC converter, is the least effective, specially when the underlying converter has low efficiency. Indeed, it dissipates on average more energy than the passive balancing, if the converter efficiency is below $50 \%$. An important conclusion of the comparison is that the type of energy transfer has an impact on the performances of an active balancing technique as significant as the efficiency of the converter used. Finally, the developed balancing models provide a useful tool to compute the balancing performances of the designed balancing circuit. For instance, if the number of shunt resistors simultaneously enabled in the Cell to Null topology is limited to half the number of cells, the balancing time increases on average by only $7 \%$, while the thermal design of the BMS is significantly relaxed as the maximum power dissipated by the balancing circuit is halved.

\section{References}

[1] L. Lu, X. Han, J. Li, J. Hua, M. Ouyang, J. Power Sources 226 (2013) $272-288$.

[2] M. Einhorn, W. Roessler, J. Fleig, IEEE Trans. Veh. Technol. 60 (2011) $2448-2457$. 
[3] L. Zhong, C. Zhang, Y. He, Z. Chen, Appl. Energy 113 (2014) 558-564.

[4] H. Rahimi-Eichi, U. Ojha, F. Baronti, M.-Y. Chow, IEEE Ind. Electron. Mag. 7 (2013) 4-16.

[5] A. Baughman, M. Ferdowsi, IEEE Trans. Ind. Electron. 55 (2008) 22772285.

[6] P. A. Cassani, S. S. Williamson, IEEE Trans. Ind. Electron. 57 (2010) 3956-3962.

[7] C.-H. Kim, M.-Y. Kim, H.-S. Park, G.-W. Moon, IEEE Trans. Power Electron. 27 (2012) 3764-3774.

[8] C.-H. Kim, M.-Y. Kim, G.-W. Moon, IEEE Trans. Power Electron. 28 (2013) 3779-3787.

[9] T. A. Stuart, W. Zhu, J. Power Sources 196 (2011) 458-464.

[10] S.-H. Park, K.-B. Park, H.-S. Kim, G.-W. Moon, M.-J. Youn, IEEE Trans. Power Electron. 27 (2012) 2900-2911.

[11] A. M. Imtiaz, F. H. Khan, IEEE Trans. Power Electron. 28 (2013) 59605975.

[12] M. Daowd, N. Omar, P. Van Den Bossche, J. Van Mierlo, in: 2011 IEEE Veh. Power Propuls. Conf., IEEE, 2011, pp. 1-7.

[13] J. Gallardo-Lozano, E. Romero-Cadaval, M. I. Milanes-Montero, M. A. Guerrero-Martinez, J. Power Sources 246 (2014) 934-949.

[14] F. Baronti, G. Fantechi, E. Leonardi, R. Roncella, R. Saletti, in: IECON 2010 - 36th Annu. Conf. IEEE Ind. Electron. Soc., IEEE, 2010, pp. 2329 2333.

[15] F. Baronti, G. Fantechi, R. Roncella, R. Saletti, IEEE Trans. Ind. Informatics 9 (2013) 1139-1147. 


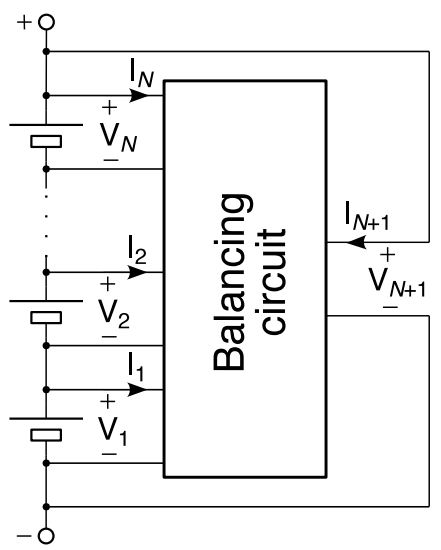

Figure 1: Model of a generic balancing circuit. 


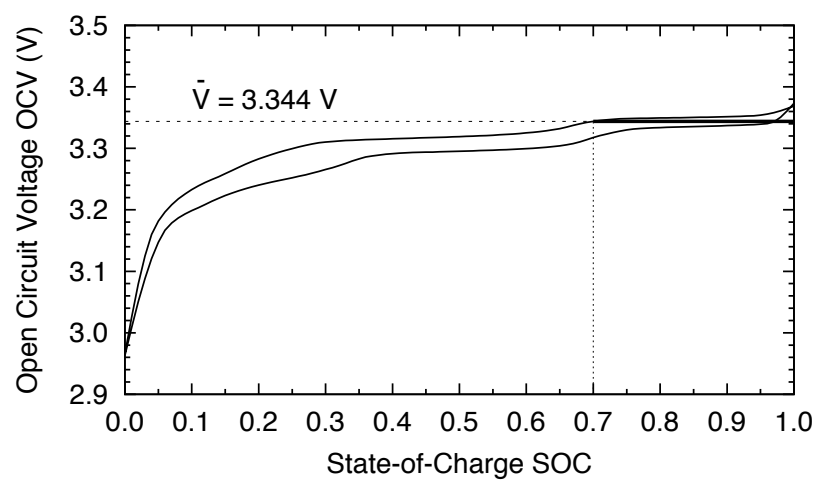

Figure 2: $S o C-O C V$ relationship measured on a $\mathrm{LiFePO}_{4}$ cell. The constant voltage approximation is well justified in the $S o C$ range above $70 \%$. 


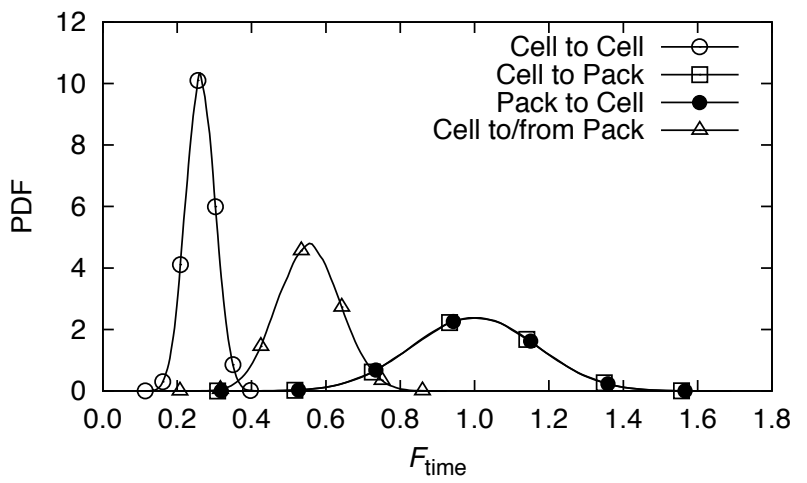

Figure 3: Probability Density Function $(\mathrm{PDF})$ of the balancing time figure $F_{\text {time }}$. 


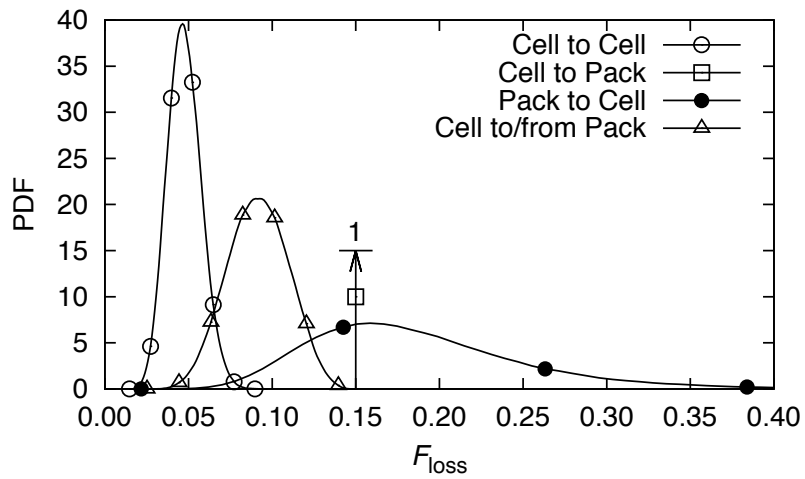

Figure 4: Probability Density Function (PDF) of the energy loss figure $F_{\text {loss }}$. 


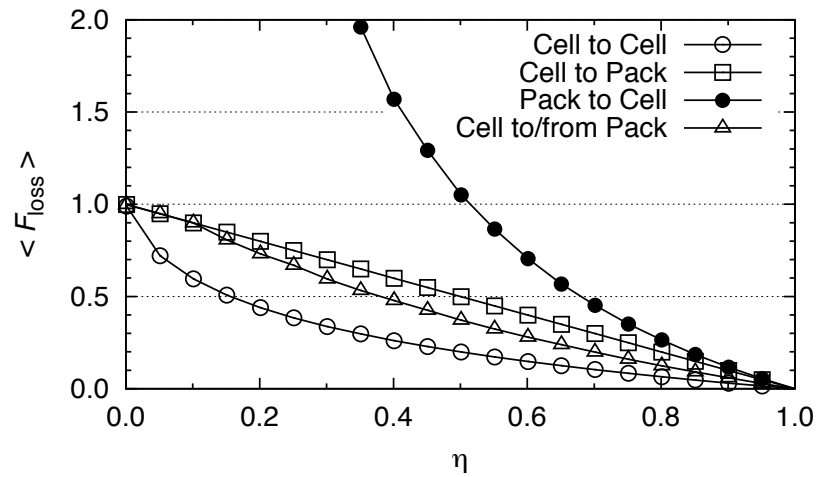

Figure 5: Mean value $<F_{\text {loss }}>$ of $F_{\text {loss }}$ versus the converter efficiency $\eta$. 


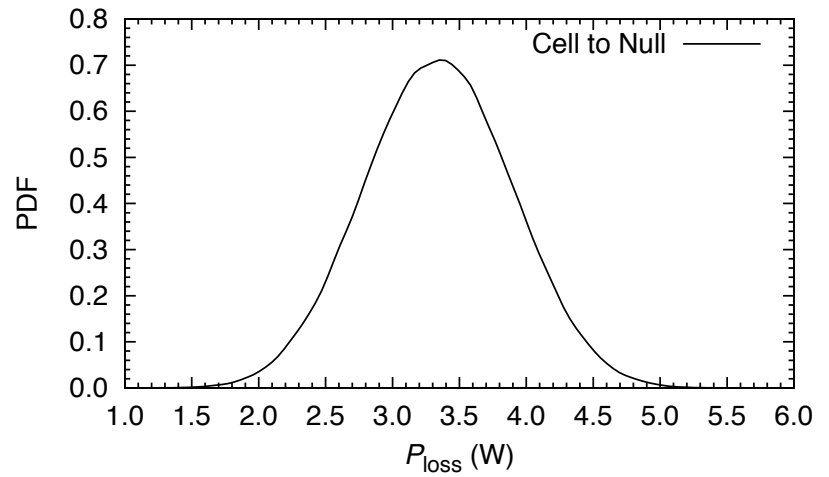

Figure 6: Probability Density Function (PDF) of $P_{\text {loss }}$ for the Cell to Null topology. 


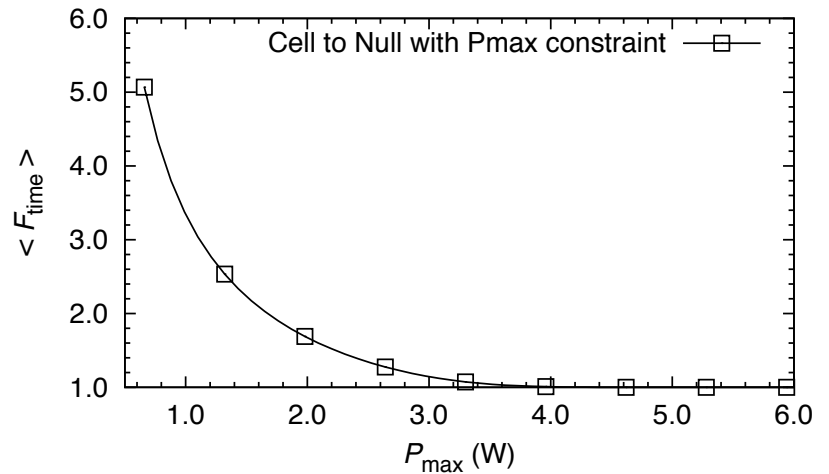

Figure 7: Trade-off between the balancing time figure and the maximum dissipated power $P_{\max }$ for the Cell to Null topology. 
Table 1: Summary of the parameters used in the comparison, if not otherwise specified.

Number of cells

$N=10$

Constant cell voltage

$\bar{V}=3.344 \mathrm{~V}$

Shunt current

$I_{\mathrm{sh}}=200 \mathrm{~mA}$

$\mathrm{DC} / \mathrm{DC}$ balancing current

DC/DC efficiency

$I_{\text {bal }}=1 \mathrm{~A}$

Maximum $S o C$ mismatch

$\eta=0.85$

$\delta=0.1$ 